\title{
RECURSIVE PROPERTIES OF ISOMORPHISM TYPES
}

\author{
MICHAEL MOSES
}

(Received 24 November 1981)

Communicated by J. N. Crossley

\begin{abstract}
For $\Gamma$ a recursively enumerable set of formulae, a structure $\mathfrak{A}$ on a recursive universe is said to be " $\Gamma$-recursively enumerable" if the satisfaction predicate restricted to $\Gamma$ is recursively enumerable (equivalently, if the formulae of $\Gamma$ uniformly denote recursively enumerable relations on $\mathfrak{A}$ ).

For recursively enumerable sets $\Gamma_{1} \subseteq \Gamma_{2}$ of formulae we shall, under certain conditions, characterize structures $\mathfrak{A}$ with the following properties.

1) Every isomorphism from $\mathfrak{A}$ to a $\Gamma_{1}$-recursively enumerable structure is a recursive isomorphism.

2) Every $\Gamma_{1}$-recursively enumerable structure isomorphic to $\mathfrak{A}$ is recursively isomorphic to $\mathfrak{A}$.

3) Every $\Gamma_{1}$-recursively enumerable structure isomorphic to $\mathfrak{A}$ is $\Gamma_{2}$-recursively enumerable.
\end{abstract}

1980 Mathematics subject classification (Amer. Math. Soc.): 03 D 45.

\section{Introduction}

A structure on a recursive universe is said to be decidable if the satisfaction predicate is recursive (equivalently, if all formulae uniformly denote recursive relations). It is said to be recursive (respectively recursively enumerable) if the satisfaction predicate restricted to atomic formulae is recursive (respectively, recursively enumerable).

Structures with certain recursive properties have been characterized algebraically. Some of these characterizations have been proved using very similar finite injury priority constructions. This paper presents the basic technique of these constructions. The concepts of recursive and decidable structures are generalized to " $\Gamma$-recursively enumerable" structures and the results presented in this context. Some of the results generalized are the characterizations of structures with the

(c) Copyright Australian Mathematical Society 1983 
following recursive properties. (The results appear in this paper as the corollaries numbered.)

(Cor. 3.1) Every isomorphism from $\mathfrak{A}$ to a recursive structure is a recursive isomorphism (Ash and Nerode [1], Goncharov [3]).

(Cor 2.2) Every recursive structure isomorphic to $\mathfrak{A}$ is recursively isomorphic to $\mathfrak{A}$; that is, $\mathfrak{A}$ is "recursively categorical" (Goncharov [3]).

(Cor. 2.1) Every decidable structure isomorphic to $\mathfrak{A}$ is recursively isomorphic to $\mathfrak{A}$; that is, $\mathfrak{A}$ is "decidably categorical" (Nurtazin [6]).

(Cor. 1.2) Every recursive structure isomorphic to $\mathfrak{A}$ is a decidable structure (Nurtazin [6]).

(Cor. 1.1) Every isomorphism from $\mathfrak{A}$ to a recursive structure carries $R$ (a relation on $\mathfrak{U}$ ) to a recursively enumerable relation; that is, $R$ is "intrinsically recursively enumerable" on $\mathfrak{A}$ (Ash and Nerode [1]).

Our general results have as corollaries some results new to the literature (for example Corollaries 1.4 and 2.3).

We consider only structures in an effective language $E$ and with recursive universes. Without loss of generality we take the non-logical symbols of $\mathcal{E}$ to be a list $\left\{P_{i}: i<\omega\right\}$ of predicates. We write $\mathfrak{A}, \mathfrak{B}$ for structures on universes $A, B$ respectively. Some model-theoretic terminology used may need to be defined.

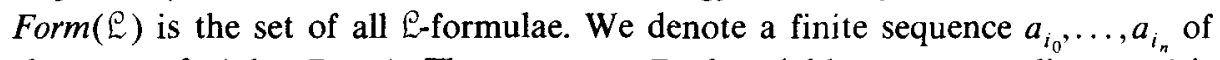
elements of $A$ by $\bar{a} \subseteq A$. The sequence $\bar{x}$ of variables corresponding to $\bar{a}$ is $\bar{x}=x_{i_{0}}, \ldots, x_{i_{n}}$. If $f$ is a function from $A$ to $B$ we write $f(\bar{a})$ for the sequence $f\left(a_{i_{0}}\right), \ldots, f\left(a_{i_{n}}\right) .\left.f\right|_{M}$ is the restriction of $f$ to $M$.

For $\Gamma \subseteq$ Form $(\mathcal{E})$ we define:

$\neg \Gamma=\{\neg \phi(\bar{x}): \phi(\bar{x}) \in \Gamma\}$,

$\wedge \Gamma=\left\{\phi_{1}\left(\bar{x}_{1}\right) \wedge \cdots \wedge \phi_{n}\left(\bar{x}_{n}\right): \phi_{i}\left(\bar{x}_{i}\right) \in \Gamma\right\}$, that is, all finite conjunctions,

$\exists \Gamma=\{\exists \bar{x} \phi(\bar{x}, \bar{y}): \phi(\bar{x}, \bar{y}) \in \Gamma\}$, and

$\forall \Gamma=\{\forall \bar{x} \phi(\bar{x}, \bar{y}): \phi(\bar{x}, \bar{y}) \in \Gamma\}$.

We say $\psi(\bar{a}, \bar{y})$ is an atom of the Lindenbaum algebra $B(\mathrm{Th}\langle\mathfrak{A}, \bar{a}\rangle)$ if for every formula $\phi(\bar{a}, \bar{y})$ if $\Re \vDash \exists \bar{y}(\psi(\bar{a}, \bar{y}) \wedge \phi(\bar{a}, \bar{y}))$ then $\mathfrak{A} \vDash \forall \bar{y}(\psi(\bar{a}, \bar{y}) \rightarrow \phi(\bar{a}, \bar{y}))$. An equivalent definition is: if $\bar{a}_{1}, \bar{a}_{2} \subseteq A$ are such that $\mathfrak{A} \vDash \psi\left(\bar{a}, \bar{a}_{1}\right)$ and $\mathfrak{A} \vDash$ $\psi\left(\bar{a}, \bar{a}_{2}\right)$, then there is an automorphism $f: \mathfrak{A} \cong \mathfrak{A}$ such that $f(\bar{a})=\bar{a}$ and $f\left(\bar{a}_{1}\right)=\bar{a}_{2}$.

\{\} is the empty set. 
Let $\Gamma$ be a recursively enumerable (r.e.) subset of Form( $\mathfrak{L}$ ). An $\mathcal{E}$-structure $\mathfrak{A}$ is $\Gamma$-recursively enumerable ( $\Gamma$-r.e.) if it has a recursive universe and the satisfaction predicate restricted to $\Gamma$ is recursively enumerable. The following are equivalent formulations of the definition.

i) The set of sentences $\{\phi(\bar{a}): \phi(\bar{x}) \in \Gamma, \bar{a} \subseteq A$ and $\mathfrak{A} \vDash \phi(\bar{a})\}$ is r.e.

ii) There is a partial effective procedure which when applied to any $\bar{a} \subseteq A$ and $\phi(\bar{x}) \in \Gamma$ terminates if and only if $\mathfrak{A} \vDash \phi(\bar{a})$.

Listed below are some examples of $\Gamma$-r.e. structures.

1) A structure $\mathfrak{A}$ with an r.e. relation $R$. $\mathfrak{A}$ is $\Gamma$-r.e. for $\Gamma=\{R\}$.

2) An r.e. structure $\mathfrak{A} . \mathfrak{A}$ is $\Gamma$-r.e. for $\Gamma=\left\{P_{i}: i<\omega\right\}$.

3) A recursive (rec.) structure $\mathfrak{A} . \mathfrak{A}$ is $\Gamma$-r.e. for $\Gamma=\left\{P_{i}: i<\omega\right\} \cup\left\{\neg P_{i}\right.$ : $i<\omega\}$.

4) A decidable (dec.) structure $\mathfrak{A}$. $\mathfrak{A}$ is $\Gamma$-r.e. for $\Gamma=$ Form( $(\mathfrak{C})$.

Notice that if $\mathfrak{A}$ is $\Gamma$-r.e. then it is also $\exists \wedge \Gamma$-r.e.

Let $\Gamma_{1} \subseteq \Gamma_{2}$ be r.e. sets of $\varrho$-formulae. Our first theorem characterizes structures $\mathfrak{A}$ with the property that every $\Gamma_{1}$-r.e. structure isomorphic to $\mathfrak{A}$ is $\Gamma_{2}$-r.e. The characterization is, however, subject to a certain decidability assumption.

For $\Sigma_{1}, \Sigma_{2} \subseteq$ Form $(\ell)$ we say $\mathfrak{A}$ is $\Sigma_{1} \rightarrow \Sigma_{2}$-decidable if there is an effective procedure which when applied to any $\bar{a} \subseteq A, \psi(\bar{x}, \bar{y}) \in \Sigma_{1}$ and $\phi(\bar{x}, \bar{y}) \in \Sigma_{2}$ determines whether or not $\mathfrak{A} \vDash \forall \bar{y}(\psi(\bar{a}, \bar{y}) \rightarrow \phi(\bar{a}, \bar{y}))$. Taking $\bar{y}$ to be the empty sequence and $\phi$ any formula such that $\mathfrak{A} \vDash \neg \phi(\bar{a})$ we see that if $\mathfrak{A}$ is $\Sigma_{1} \rightarrow \Sigma_{2}$-dec. it is $\Sigma_{1}$-rec. (that is, $\Sigma_{1} \cup \neg \Sigma_{1}$-r.e.). Similarly, taking $\psi$ to be any formula such that $\mathfrak{A} \vDash \psi(\bar{a})$, we see that $\mathfrak{A}$ is $\Sigma_{2}$-rec. We may concern ourselves only with sets $\Sigma_{1}$ and $\Sigma_{2}$ for which such formulae $\psi$ and $\phi$ exist because the results in which we use this definition (Theorems I, II and III) are otherwise trivially true.

THeOREM I. Let $\Gamma_{1}, \Gamma_{2}$ be r.e. sets with $\left\{P_{i}: i<\omega\right\} \subseteq \Gamma_{1} \subseteq \Gamma_{2} \subseteq$ Form( $(\varrho)$ and $\exists \wedge \Gamma_{1}$ rec. in $\Gamma_{2}$. If $\mathfrak{A}$ is $\left(\exists \wedge \Gamma_{1}\right) \rightarrow\left(\Gamma_{2}-\left(\exists \wedge \Gamma_{1}\right)\right)$-dec, the following are equivalent.

1) Every $\Gamma_{1}$-r.e. structure isomorphic to $\mathfrak{A}$ is $\Gamma_{2}$-r.e.

2) For some $\bar{a} \subseteq A$ there is an effective procedure which when applied to any $\bar{b} \subseteq A-\bar{a}$ and $\Gamma_{2}$-formula $\phi(\bar{x}, \bar{y})$ such that $\mathfrak{A} \vDash \phi(\bar{a}, \bar{b})$, produces a $\exists \wedge \Gamma_{1^{-}}$ formula $\psi(\bar{x}, \bar{y})$ such that $\mathfrak{A} \vDash \psi(\bar{a}, \bar{b})$ and $\mathfrak{A} \vDash \forall \bar{y}(\psi(\bar{a}, \bar{y}) \rightarrow \phi(\bar{a}, \bar{y}))$.

Proof. We first show that 2$) \Rightarrow 1$ ). Let $\mathfrak{B}$ be a $\Gamma_{1}$-r.e. structure and $f: \mathfrak{A} \cong \mathfrak{B}$. We show that $B$ is $\Gamma_{2}$-r.e.

Let $\bar{a}$ be the sequence described in 2) and $\left\{\phi_{1}\left(\bar{a}, \bar{a}_{1}\right), \Phi_{2}\left(\bar{a}, \bar{a}_{2}\right), \ldots\right\}$ an effective listing of all $\Gamma_{2}$-sentences true in $\mathfrak{U}\left(\mathfrak{U}\right.$ is $\Gamma_{2}$-r.e.). Apply the effective 
procedure described in 2) to each element of this list to obtain another effective list $\left\{\psi_{1}\left(\bar{a}, \bar{y}_{1}\right), \psi_{2}\left(\bar{a}, \bar{y}_{2}\right), \ldots\right\}$ of formulae such that $\forall i<\omega, \mathfrak{A} \vDash \psi_{i}\left(\bar{a}, \bar{a}_{i}\right)$ and $\mathfrak{A} \vDash \forall \bar{y}_{i}\left(\psi_{i}\left(\bar{a}, \bar{y}_{i}\right) \rightarrow \phi_{i}\left(\bar{a}, \bar{y}_{i}\right)\right) . \mathfrak{B}$ is $\Gamma_{1}$-r.e. and therefore $\exists \wedge \Gamma_{1}$-r.e. Thus there is an effective list of all $\exists \wedge \Gamma_{1}$-sentences $\phi(\bar{b})$ true in $\mathscr{B}$. If for some $i<\omega$ and $\bar{b} \subseteq B \quad \psi_{i}(f(\bar{a}), \bar{b})$ is a member of this list, then list $\phi_{i}(f(\bar{a}), \bar{b})$ as true in $\mathfrak{B}$. We show that this (effective) process lists precisely those $\Gamma_{2}$-sentences true in $\mathfrak{B}$.

If the process decides $\phi_{i}(f(\bar{a}), \bar{b})$ is true in $\mathscr{B}$, then $\mathscr{B} \vDash \psi_{i}(f(\bar{a}), \bar{b})$. But $\mathfrak{U} \vDash \forall \bar{y}_{i}\left(\psi_{i}\left(\bar{a}, \bar{y}_{i}\right) \rightarrow \phi_{i}\left(\bar{a}, \bar{y}_{i}\right)\right)$ and $f: \mathfrak{U} \cong \mathfrak{B}$. Therefore $\mathfrak{B} \vDash \forall \bar{y}_{i}\left(\psi_{i}\left(f(\bar{a}), \bar{y}_{i}\right) \rightarrow\right.$ $\left.\phi_{i}\left(f(\bar{a}), \bar{y}_{i}\right)\right)$; and so $\mathfrak{B} \vDash \phi_{i}(f(\bar{a}), \bar{b})$.

If $\phi(f(\bar{a}), \bar{b})$ is a $\Gamma_{2}$-sentence true in $\mathfrak{B}$, then, since $f: \mathfrak{A} \cong \mathscr{B}, \mathfrak{A} \vDash \phi\left(\bar{a}, f^{-1}(\bar{b})\right)$. Therefore, since $\mathfrak{A}$ is $\Gamma_{2}$-r.e., for some $i<\omega, \phi\left(\bar{a}, f^{-1}(\bar{b})\right)=\phi_{i}$. Then $\mathfrak{A} F$ $\psi_{i}\left(\bar{a}, f^{-1}(\bar{b})\right)$ and, as before, we have $\mathfrak{B} \vDash \psi_{i}(f(\bar{a}), \bar{b})$. Thus, the process will list $\phi(f(\bar{a}), \bar{b})$ as true in $\mathfrak{B}$. This shows that $\mathfrak{B}$ is $\Gamma_{2}$-r.e.

We now show that $\neg 2) \Rightarrow \neg 1$ ) via a finite injury priority construction. Let $\mathfrak{A}$ be a $\left(\exists \wedge \Gamma_{1}\right) \rightarrow\left(\Gamma_{2}-\left(\exists \wedge \Gamma_{1}\right)\right)$-dec. structure on recursive universe $A=$ $\left\{a_{0}, a_{1}, \ldots\right\}$ satisfying $\neg 2$ ). We shall construct a $\Gamma_{1}$-r.e. $\mathscr{B}$ in stages and a $g: \mathfrak{B} \cong \mathfrak{A}$ such that $\mathfrak{B}$ is not $\Gamma_{2}$-r.e., thus showing that 1$)$ is false. $\mathfrak{B}$ will be a structure on recursive universe $B=\left\{b_{0}, b_{1}, \ldots\right\}$.

At each stage $s$ of our construction we shall define a partial map $g_{s}: B \rightarrow A$ so that $g=\lim _{s} g_{s}$ exists, and is a surjection from $B$ to $A$. At stage $s$ we define a finite set $\Sigma^{s}$ of $\Gamma_{1}$-sentences $\phi(\bar{b})$ as follows. Since $\mathfrak{A}$ is $\Gamma_{1}$-r.e. there is an effective list of all $\Gamma_{1}$-sentences $\phi(\bar{a})$ true in $\mathfrak{A}$. We define $\Sigma^{-1}=\{\}$ and $\Sigma^{s}=\Sigma^{s-1} U$ $\left\{\phi(\bar{b}): \phi(\bar{x}) \in \Gamma, \bar{b} \subseteq \operatorname{dom}\left(g_{s}\right)\right.$ and $\phi\left(g_{s}(\bar{b})\right)$ has been listed as true in $\mathfrak{A}$ by this stage\}. We write $\wedge \Sigma^{s}$ for the conjunction of all the sentences in $\Sigma^{s} . \mathfrak{B}$ is defined to be the structure on $B$ satisfying all the quantifier-free sentences in $\Sigma=\cup_{s} \Sigma^{s}$. We will ensure that $g: \mathfrak{B} \cong \mathfrak{A}$.

Let $W_{0}, W_{1}, \ldots$ be a list of all r.e. subsets of $\Gamma_{2}$-sentences $\phi(\bar{b})$ with $\bar{b} \subseteq B$. The idea of the construction is to use the hypothesis to diagonalize over the $W_{e}: e<\omega$ to ensure that none of them lists precisely those $\Gamma_{2}$-sentences true in $\mathfrak{B} . W_{e}^{s}$ denotes the subset of $W_{e}$ calculated by stage $s$.

Our requirements are:

$P_{e}^{1}: b_{e} \in \operatorname{dom}(g)$,

$P_{e}^{2}: a_{e} \in \operatorname{ran}(g)$, and

$Q_{e}: W_{e} \neq\left\{\phi(\bar{b}): \phi(\bar{x}) \in \Gamma_{2}, \bar{b} \subseteq B\right.$ and $\left.\mathfrak{B} \vDash \phi(\bar{b})\right\}$.

The basic action to meet the requirements $Q_{e}$ is this. If at stage $s$ we have $\phi(\bar{b}) \in W_{e}^{s}$ with $\bar{b} \subseteq \operatorname{dom}\left(g_{s}\right)$, at stage $s+1$ we attempt to define $g_{s+1}$ so that $\mathfrak{U} \vDash \neg \phi\left(g_{s+1}(\bar{b})\right)$; and therefore $\mathfrak{B} \vDash \neg \phi(\bar{b})$. A problem arising from this action is that since $g_{s+1}(\bar{b}) \neq g_{s}(\bar{b})$, we run the risk that $g=\lim _{s} g_{s}$ may not exist. Another problem is that as we want $\Sigma=\cup_{s} \Sigma^{s}$ to be the $\Gamma_{1}$-diagram of $\mathfrak{B}$, we must arrange that no sentence of $\Sigma^{s}$ is made false by our definition of $g_{s+1}$. 
Definitions. $P_{e}^{1}$ requires attention at stage $s+1$ if $b_{e} \notin \operatorname{dom}\left(g_{s}\right)$.

$P_{e}^{1}$ is injured at stage $s+1$ if $g_{s+1}\left(b_{e}\right) \neq g_{s}\left(b_{e}\right)$,

$P_{e}^{2}$ requires attention at stage $s+1$ if $a_{e} \notin \operatorname{ran}\left(g_{s}\right)$.

$P_{e}^{2}$ is injured at stage $s+1$ if $g_{s+1}^{-1}\left(a_{e}\right) \neq g_{s}^{-1}\left(a_{e}\right)$.

In order to meet the requirements $Q_{e}$ we define at some stages $s$ sequences $\bar{d}_{e}^{s} \subseteq B$ with the intention that if $\left\{\phi(\bar{b}): \phi(\bar{x}) \in \Gamma_{2}, \bar{b} \subseteq B\right.$ and $\left.\mathfrak{B} \vDash \phi(\bar{b})\right\} \subseteq W_{e}$, then $\bar{d}_{e}=\lim _{s} \bar{d}_{e}^{s}$ exists, and there is a formula $\theta(\bar{x}) \in \Gamma_{2}$ such that $\theta\left(\bar{d}_{e}\right) \in W_{e}$, but $\mathscr{B} \forall \theta\left(\bar{d}_{e}\right)$.

At any stage $s$ when $P_{0}^{1}, \ldots, P_{e}^{1}, P_{0}^{2}, \ldots, P_{e}^{2}$ do not require attention we define for $\bar{b} \subseteq \operatorname{dom}\left(g_{s}\right)$ the splitting of $\operatorname{dom}\left(g_{s}\right)$ with respect to $Q_{e}$ and $\bar{b}$ to be $\operatorname{dom}\left(g_{s}\right)=\bar{b}_{0}, \bar{b}_{1}, \bar{b}_{2}$ where:

$\bar{b}_{0}=b_{0}, b_{1}, \ldots, b_{e}, g_{s}^{-1}\left(a_{0}\right), \ldots, g_{s}^{-1}\left(a_{e}\right), \cup \overline{d_{e^{\prime}}^{s}}$ with the union taken over all $e^{\prime}<e$ for which $\overline{d_{e^{\prime}}^{s}}$ is defined,

$\bar{b}_{1}=\bar{b}-\bar{b}_{0}$, and

$\bar{b}_{2}=\operatorname{dom}\left(g_{s}\right)-\left(\bar{b}_{0} \cup \bar{b}_{1}\right)$.

A sentence $\phi(\vec{b}) \in W_{e}^{s+1}$ can be used to attack $Q_{e}$ at stage $s+1$ if

1) $\bar{b} \subseteq \operatorname{dom}\left(g_{s}\right)$

2) $\phi(\bar{x}) \notin \exists \wedge \Gamma_{1}$, and

3) $\mathfrak{A} \vDash \exists \bar{x}_{1}\left(\exists \bar{x}_{2} \wedge \Sigma^{s}\left(g_{s}\left(\bar{b}_{0}\right), \bar{x}_{1}, \bar{x}_{2}\right) \wedge \neg \phi\left(g_{s}\left(\bar{b}_{0}\right), \bar{x}_{1}\right)\right)$ where $\bar{b}_{0}, \bar{x}_{1}, \bar{x}_{2}$ correspond to $\bar{b}_{0}, \bar{b}_{1}, \bar{b}_{2}$ in the splitting of $\operatorname{dom}\left(g_{s}\right)$ with respect to $Q_{e}$ and $\vec{b}$.

$Q_{e}$ is injured at stage $s+1$ if $g_{s+1}\left(\overline{d_{e}^{s}}\right) \neq g_{s}\left(\overline{d_{e}^{s}}\right)$. In this case we say that $\overline{d_{e}^{s+1}}$ is undefined. (Otherwise $\overline{d_{e}^{s+1}}=\overline{d_{e}^{s}}$ )

Construction. Stage 0. Define $g_{0}: b_{0} \rightarrow a_{0}$.

Stage $s+1$. Arrange the requirements in order of decreasing priority as $P_{0}^{1}, P_{0}^{2}$, $Q_{0}, P_{1}^{1}, P_{1}^{2}, Q_{1}, \ldots$ and look for the one of highest priority requiring attention at this stage. Attack the requirement as follows.

If the requirement is a $P_{e}^{1}$, attack it by defining $g_{s+1}\left(b_{e}\right)$ to be the least $a_{n} \notin \operatorname{ran}\left(g_{s}\right) ;$ and $\left.g_{s+1}\right|_{\text {dom }\left(g_{s}\right)}=g_{s}$.

If the requirement is a $P_{e}^{2}$, attack it by defining $g_{s+1}^{-1}\left(a_{e}\right)$ to be the least $b_{n} \notin \operatorname{dom}\left(g_{s}\right)$; and $\left.g_{s+1}\right|_{\operatorname{dom}\left(g_{s}\right)}=g_{s}$.

If the requirement is a $Q_{e}$, we choose the first $\phi(\bar{b}) \in W_{e}^{s+1}$ which can be used to attack $Q_{e}$ at this stage and define $g_{s+1}$ as follows.

$g_{s+1}\left(\bar{b}_{0}\right)=g_{s}\left(\bar{b}_{0}\right)$.

$g_{s+1}\left(\bar{b}_{1}\right)=$ the least sequence $\bar{a}_{1}$ disjoint from $g_{s}\left(\bar{b}_{0}\right)$ such that $\mathfrak{U} \vDash \exists \bar{x}_{2} \wedge$ $\Sigma^{s}\left(g_{s}\left(\bar{b}_{0}\right), \bar{a}_{1}, \bar{x}_{2}\right) \wedge \neg \phi\left(g_{s}\left(\bar{b}_{0}\right), \bar{a}_{1}\right)$. There is such an $\bar{a}_{1}$ by property 3 of $\phi(\bar{b})$.

$g_{s+1}\left(\bar{b}_{2}\right)=$ the least sequence $\bar{a}_{2}$ disjoint from $g_{s}\left(\bar{b}_{0}\right) \cup \bar{a}_{1}$ such that $\mathfrak{A} F$ $\wedge \Sigma^{s}\left(g_{s}\left(\bar{b}_{0}\right), \bar{a}_{1}, \bar{a}_{2}\right)$. There is such an $\bar{a}_{2}$ by definition of $\bar{a}_{1}$. Define $\overline{d_{e}^{s+1}}=\bar{b}$.

We conclude the proof with the following remarks. 
1) The construction is effective. The only problem is to decide when and how to attack a requirement $Q_{e}$. At stage $s+1$ we only need consider whether or not one of the (finite number of) requirements $Q_{e}$ with $e \leqslant s+1$ requires attention. To do this we run through the (finite number of) sentences in $W_{e}^{s+1}$ asking if there are any that can be used to attack $Q_{e}$ at this stage. This can be done effectively since $\mathfrak{A}$ is $\left(\exists \wedge \Gamma_{1}\right) \rightarrow\left(\Gamma_{2}-\left(\exists \wedge \Gamma_{1}\right)\right)$-decidable.

Once we have decided to attack a requirement $Q_{e}$, we need to find the sequences $\bar{a}_{1}, \bar{a}_{2}$. To do this we run through all sequences of the right length from $A$ until we find sequences that fit the bill. Once again the decidability assumption on $\mathfrak{A}$ allows us to do this effectively.

2) Each requirement $Q_{e}$ is attacked at most a finite number of times. Assume this to be true for the requirements $Q_{0}, \ldots, Q_{e-1}$. We show it to be true for $Q_{e}$. Let $t$ be a stage after which none of $Q_{0}, \ldots, Q_{e-1}$ are ever attacked. Then by the fact that $\cup \overline{d_{e}^{s}} \subseteq b_{0}$ in the definition of the splitting of $\operatorname{dom}\left(g^{s}\right), Q_{e}$ is never injured after this stage. Thus $Q_{e}$ is attacked at most once after stage $t$.

LEMMA 1. $g=\lim _{s} g_{s}$ exists, and $g: \mathfrak{B} \cong \mathfrak{A}$.

Proof. We show that for any $e<\omega, g$ is re-defined on $b_{e}$ and $a_{e}$ at most a finite number of times; and therefore $\lim _{s} g_{s}\left(b_{e}\right)$ and $\lim _{s} g_{s}^{-1}\left(a_{e}\right)$ exist. Let $t$ be a stage after which none of $Q_{0}, \ldots, Q_{e^{-1}}$ are ever attacked. Then, as in the last proof, since $b_{e}, g_{s}^{-1}\left(a_{e}\right) \in \bar{b}_{0}, P_{e}^{1}$ and $P_{e}^{2}$ are never injured after this stage. Thus $\lim _{s} g_{s}\left(b_{e}\right)=g_{t}\left(b_{e}\right)$ and $\lim _{s} g_{s}^{-1}\left(a_{e}\right)=g_{t}^{-1}\left(a_{e}\right)$. Therefore $g=\lim _{s} g_{s}$ exists.

Since for any $e<\omega, P_{e}^{1}$ and $P_{e}^{2}$ are attacked at some stage; $g: B \rightarrow A$ is a surjection.

We now show that for every predicate $P$ in $\varrho, B F P(\bar{b})$ if and only if $\mathfrak{A} \vDash P(g(\bar{b}))$.

Let $\mathfrak{A} \vDash P(\bar{b})$. Then, by definition of $\mathfrak{B}, P(\bar{b}) \in \Sigma$; that is, $P(\bar{b}) \in \Sigma^{s}-\Sigma^{s-1}$ for some $s$. By definition of $\Sigma^{s}, \mathfrak{A} \vDash P\left(g_{s}(\bar{b})\right)$. If at some further stage $t+1$ we re-define $g$ on $\bar{b}$, we make sure that $\mathfrak{A} \vDash \wedge \Sigma^{\prime}\left(g_{t+1}\left(\operatorname{dom}\left(g_{t}\right)\right)\right)$. Therefore $\mathfrak{A} \vDash$ $P\left(g_{t+1}(\bar{b})\right)$. Thus $\mathfrak{A} \vDash P(g(\bar{b}))$.

Let $\mathfrak{A} \vDash P(g(\bar{b}))$. Let $s$ be a stage when $g$ has taken on its final value on $\bar{b}$. Since $\mathfrak{A}$ is $\Gamma_{1}$-r.e., there is a stage $t(\geqslant s)$ by which $P(g(\bar{b}))$ has been listed as true in $\mathfrak{A}$. Then, by definition of $\Sigma^{t}, P(\bar{b}) \in \Sigma^{t} \subseteq \Sigma$. Thus $\mathfrak{B} \vDash P(\bar{b})$.

LEMMA 2. $\mathscr{B}$ is a $\Gamma_{1}$-r.e. structure.

Proof. By applying to any $\Gamma_{1}$-formula $\phi$ the argument applied to the predicate $P$ in Lemma 1 , we see that $\phi(\bar{b}) \in \Sigma$ if and only if $\mathscr{H} \vDash \phi(g(\bar{b})$ ). Since $g: \mathfrak{B} \cong \mathfrak{A}$, 
we therefore have $\Sigma=\left\{\phi(\bar{b}): \phi(\bar{x}) \in \Gamma_{1}, \bar{b} \subseteq B\right.$ and $\left.\mathscr{B} \vDash \phi(\bar{b})\right\}$. $\Sigma$ is r.e. (by Remark 1), and therefore $\mathfrak{B}$ is $\Gamma_{1}$-r.e.

LEMMA 3. $\mathfrak{B}$ is not $\Gamma_{2}-$ r.e.

Proof. Suppose $\mathfrak{B}$ is $\Gamma_{2}$-r.e. We show that statement 2) of the theorem holds. Let $e$ be least with $W_{e}=\left\{\phi(\bar{b}): \phi(\bar{x}) \in \Gamma_{2}, \bar{b} \subseteq B\right.$ and $\left.\mathscr{B} \vDash \phi(\bar{b})\right\}$. Consider a stage $s_{0}$ after which none of $Q_{0}, \ldots, Q_{e-1}$ are ever attacked and $P_{0}^{1}, \ldots, P_{e}^{1}$, $P_{0}^{2}, \ldots, P_{e}^{2}$ do not require attention.

$Q_{e}$ is not injured after stage $s_{0}$. Suppose $Q_{e}$ were attacked at some stage $t \geqslant s_{0}$ by a sentence $\phi(\bar{b})$. Then $\bar{b}=\bar{d}_{e}^{t}$ and as $Q_{e}$ is never injured, $g_{t}(\bar{b})=g(\bar{b})$. Thus $\phi(\bar{b}) \in W_{e}$ and $\mathfrak{A} \vDash \neg \phi(g(\bar{b}))$. Therefore, since $g: \mathfrak{B} \cong \mathfrak{A}, \mathfrak{B} \vDash \neg \phi(\bar{b})$. This contradicts the choice of $W_{e}$. Thus $Q_{e}$ is not attacked after stage $s_{0}$.

Let $\bar{b}_{0}$ be as in the splitting of $\operatorname{dom}\left(g_{s_{0}}\right)$ with respect to $Q_{e}$. By the choice of $s_{0}$, $\bar{b}_{0}$ is the same in the splitting of $\operatorname{dom}\left(g_{s}\right)$ with respect to $Q_{e}$ for any $s \geqslant s_{0}$. Therefore $g_{s_{0}}\left(\bar{b}_{0}\right)=g\left(\bar{b}_{0}\right)=\bar{a}_{0}$, say.

Let $\phi\left(\bar{b}_{0}, \bar{b}\right)$ be a $\Gamma_{2}$-sentence true in $\mathscr{B}$. We show that there is a $\exists \wedge \Gamma_{1}$-formula $\psi\left(\bar{x}_{0}, \bar{x}\right)$ such that $\mathfrak{B} \vDash \psi\left(\bar{b}_{0}, \bar{b}\right)$ and $\mathfrak{A} \vDash \forall \bar{x}\left(\psi\left(\bar{a}_{0}, \bar{x}\right) \rightarrow \phi\left(\bar{a}_{0}, \bar{x}\right)\right)$. If $\phi\left(\bar{b}_{0}, \bar{b}\right)$ is a $\exists \wedge \Gamma_{1}$-sentence, take $\psi\left(\bar{x}_{0}, \bar{x}\right)=\phi\left(\bar{x}_{0}, \bar{x}\right)$ where $\bar{x}_{0}, \bar{x}$ correspond to $\bar{b}_{0}, \bar{b}$. This clearly fits the bill.

If $\phi\left(\overline{b_{0}}, \bar{b}\right)$ is a $\Gamma_{2}-\left(\exists \wedge \Gamma_{1}\right)$-sentence, consider a stage $s \geqslant s_{0}$ of our construction when

i) $\phi\left(\bar{b}_{0}, \bar{b}\right) \in W_{e}^{s}$ (such a stage exists by choice of $W_{e}$ ), and

ii) $\bar{b} \subseteq \operatorname{dom}\left(g_{s}\right)$.

Take $\psi\left(\bar{x}_{0}, \bar{x}\right)=\exists \bar{x}_{2} \wedge \Sigma^{s}\left(\bar{x}_{0}, \bar{x}, \bar{x}_{2}\right)$, where $\bar{x}_{0}, \bar{x}, \bar{x}_{2}$ correspond to $\bar{b}_{0}, \bar{b}_{1}, \bar{b}_{2}$ in the splitting of $\operatorname{dom}\left(g_{s}\right)$ with respect to $Q_{e}$ and $\bar{b}$. Clearly $\mathfrak{B} \vDash \psi\left(\bar{b}_{0}, \bar{b}\right)$. We show that $\mathfrak{A} \vDash \forall \bar{x}\left(\psi\left(\bar{a}_{0}, \bar{x}\right) \rightarrow \phi\left(\bar{a}_{0}, \bar{x}\right)\right)$. Since $s \geqslant s_{0}, Q_{e}$ is not attacked at this stage. In particular $\phi\left(\bar{b}_{0}, \bar{b}\right)$ cannot be used to attack $Q_{e}$ at this stage. By i) and ii) above, this means that $\phi\left(\bar{b}_{0}, \bar{b}\right)$ does not satisfy property 3 ; that is, $\mathfrak{A} \vDash$ $\forall \bar{x}\left(\psi\left(\bar{a}_{0}, \bar{x}\right) \rightarrow \phi\left(\bar{a}_{0}, \bar{x}\right)\right)$.

Thus for every $\Gamma_{2}$-sentence $\phi\left(\bar{b}_{0}, \bar{b}\right)$ true in $\mathscr{B}$, there is a $\exists \wedge \Gamma_{1}$-formula $\psi\left(\bar{x}_{0}, \bar{x}\right)$ such that $\mathscr{B} \vDash \psi\left(\bar{b}_{0}, \bar{b}\right)$ and $\mathfrak{A} \vDash \forall \bar{x}\left(\psi\left(\bar{a}_{0}, \bar{x}\right) \rightarrow \phi\left(\bar{a}_{0}, \bar{x}\right)\right)$. Therefore, for every $\Gamma_{2}$-sentence $\phi\left(\bar{a}_{0}, \bar{a}\right)$ true in $\mathfrak{A}$, there is a $\exists \wedge \Gamma_{1}$-formula $\psi\left(\bar{x}_{0}, \bar{x}\right)$ such that $\mathfrak{A} \vDash \psi\left(\bar{a}_{0}, \bar{a}\right)$ and $\mathfrak{A} \vDash \forall \bar{x}\left(\psi\left(\bar{a}_{0}, \bar{x}\right) \rightarrow \phi\left(\bar{a}_{0}, \bar{x}\right)\right)$; namely the formula corresponding to $\phi\left(\bar{b}_{0}, g^{-1}(\bar{a})\right)$. Given any $\phi\left(\bar{a}_{0}, \bar{a}\right)$ true in $\mathfrak{A}$, we can find this $\exists \wedge \Gamma_{1}$-formula effectively using the fact that $\mathfrak{A}$ is $\left(\exists \wedge \Gamma_{1}\right) \rightarrow\left(\Gamma_{2}-\left(\exists \wedge \Gamma_{1}\right)\right)$ decidable.

This contradicts our hypothesis, thus proving Theorem I.

In Theorem I, $\Gamma_{2}$ was taken to be a set of formulae to simplify notation. The only property used is that every $\phi \in \Gamma_{2}$ is preserved under isomorphisms; that is, 
if $f: \mathfrak{B} \cong \mathfrak{A}$ then $\mathfrak{B} \vDash \phi(\bar{b})$ if and only if $\mathfrak{A} \vDash \phi(f(\bar{b}))$. Any relation $R$ on $\mathfrak{A}$ may be treated as such a formula by interpreting it in any $\mathfrak{B}$ with $f: \mathfrak{B} \cong \mathfrak{A}$ as the relation $f^{-1} R=\left\{f^{-1}(\bar{a}): \bar{a} \in R\right\}$. We therefore have the following result.

TheOrem I'. Let $\Gamma_{1}$ be an r.e. set with $\left\{P_{i}: i<\omega\right\} \subseteq \Gamma_{1} \subseteq$ Form( $(\varrho)$, and $\Gamma_{2}$ a set $\left\{R_{i}: i<\omega\right\}$ of relations on $\mathfrak{A}$. If $\mathfrak{A}$ is $\left(\exists \wedge \Gamma_{1}\right) \rightarrow \Gamma_{2}$-dec., the following are equivalent.

1) For every $\Gamma_{1}-r$.e. structure $\mathfrak{B}$ and isomorphism $f: \mathfrak{A} \cong \mathfrak{B}$, the relations $\left\{f R_{i}\right\}$ are uniformly r.e. on $\mathfrak{B}$.

2) For some $\bar{a} \subseteq A$ there is an effective procedure which when applied to any $\bar{b} \subseteq A-\bar{a}$ and $R_{i} \in \Gamma_{2}$ such that $\mathfrak{U} \vDash R_{i}(\bar{a}, \bar{b})$ produces $a \exists \wedge \Gamma_{1}$-formula $\psi(\bar{x}, \bar{y})$ such that $\mathfrak{A} \vDash \psi(\bar{a}, \bar{b})$ and $\mathfrak{A} \vDash \forall \bar{y}\left(\psi(\bar{a}, \bar{y}) \rightarrow R_{i}(\bar{a}, \bar{y})\right)$.

Consider a language $\mathfrak{E}$ with a predicate $\equiv$ interpreted in $\mathfrak{A}$ as the identity relation. Then the sentences $\forall \bar{y}(\psi(\bar{a}, \bar{y}) \rightarrow \phi(\bar{a}, \bar{y}))$ and $\forall \bar{y}, \bar{z}(\psi(\bar{a}, \bar{y}) \wedge(\bar{a} \equiv \bar{z})$ $\rightarrow \phi(\bar{z}, \bar{y}))$ are equivalent in $\mathfrak{A}$. We may therefore replace $\phi(\bar{a}, \bar{y})$ by $\phi(\bar{y})$ throughout the definition of $\Sigma_{1} \rightarrow \Sigma_{2}$-decidability and the statement of the theorem.

In the case $\Gamma_{1}=\left\{P_{i}: i<\omega\right\} \cup\left\{\neg P_{i}: i<\omega\right\}$ and $\Gamma_{2}=\Gamma_{1} \cup\{R\}$ Theorem I' reads as follows.

COROLlaRY 1.1 (Ash and Nerode [1]). Let $\mathfrak{A}$ be a structure with predicate $\equiv$ interpreted as the identity; and $R$ a relation on $\mathfrak{A}$. If there is an effective procedure for determining, given any existential formula $\psi(\bar{x}, \bar{y})$ and $\bar{a} \subseteq A$, whether the implication $\psi(\bar{a}, \bar{x}) \rightarrow R(\bar{x})$ is true in $\mathfrak{A}$, the following are equivalent.

1) $R$ is intrinsically r.e. on $\Re$.

2) For some $\bar{a} \subseteq A$ there is an effective procedure which, when applied to any $\bar{b} \subseteq A-\bar{a}$ such that $\mathfrak{A} \vDash R(\bar{b})$, produces an existential formula $\psi(\bar{x}, \bar{y})$ such that $\mathfrak{A} \vDash \psi(\bar{a}, \bar{b})$ and $\mathfrak{A} \vDash \forall \bar{y}(\psi(\bar{a}, \bar{y}) \rightarrow R(\bar{y}))$.

Applying Theorem I to the sets $\Gamma_{1}=\left\{P_{i}: i<\omega\right\} \cup\left\{\neg P_{i}: i<\omega\right\}$ and $\Gamma_{2}=$ Form(e) we get

Corollary 1.2 (Nurtazin [6]). For a dec. structure $\mathfrak{A}$ the following are equivalent.

1) Every recursive structure isomorphic to $\mathfrak{A}$ is decidable.

2) For some $\bar{a} \subseteq A$ there is an effective procedure which, when applied to any $\bar{b} \subseteq A-\bar{a}$ and formula $\phi(\bar{x}, \bar{y})$ such that $\mathfrak{A} \vDash \phi(\bar{a}, \bar{b})$, produces an existential formula $\psi(\bar{x}, \bar{y})$ such that $\mathfrak{U} \vDash \psi(\bar{a}, \bar{b})$ and $\mathfrak{A} \vDash \forall \bar{y}(\psi(\bar{a}, \bar{y}) \rightarrow \phi(\bar{a}, \bar{y}))$. 
A structure is n-recursive if it is $\Gamma$-r.e. for $\Gamma$ the set of all formulae with $n$ or less alternations of quantifiers; that is, of the form $Q_{1} \bar{x}_{1} Q_{2} \bar{x}_{2} \cdots Q_{m} \bar{x}_{m} \phi\left(\bar{x}_{1}, \ldots, \bar{x}_{m}\right)$ with $m \leqslant n, \phi$ quantifier-free and each $Q_{i}$ one of $\forall, \exists$.

Taking $\Gamma_{1}$ to be this $\Gamma$ and $\Gamma_{2}=\forall \Gamma_{1}$ Theorem I gives

COROLlaRY 1.3 (Goncharov [3]). For an $n+1$-rec. structure $\mathfrak{A}$, the following are equivalent.

1) Every n-rec. structure isomorphic to $\mathfrak{A}$ is $n+1-r e c$.

2) For some $\bar{a} \subseteq A$ there is an effective procedure which, when applied to any $\bar{b} \subseteq A-\bar{a}$ and $\forall \Gamma_{1}$-formula $\phi(\bar{x}, \bar{y})$ such that $\mathfrak{A} \vDash \phi(\bar{a}, \bar{b})$, produces a $\exists \Gamma_{1}$-formula $\psi(\bar{x}, \bar{y})$ such that $\mathfrak{A} \vDash \psi(\bar{a}, \bar{b})$ and $\mathfrak{A} \vDash \forall \bar{y}(\psi(\bar{a}, \bar{y}) \rightarrow \phi(\bar{a}, \bar{y}))$.

The next corollary has not appeared in the literature. For $\Gamma_{1}=\left\{P_{i}: i<\omega\right\}$ and $\Gamma_{2}=\Gamma_{1} \cup \neg \Gamma_{1}$ the theorem gives

COROLlaRY 1.4. For $a \exists \wedge \Gamma_{1}$-rec. structure $\mathfrak{A}$ the following are equivalent.

1) Every r.e. structure isomorphic to $\mathfrak{A}$ is rec.

2) For some $\bar{a} \subseteq A$ there is an effective procedure which, when applied to any $\bar{b} \subseteq A-\bar{a}$ and quantifier-free formula $\phi(\bar{x}, \bar{y})$ such that $\mathfrak{A} \vDash \phi(\bar{a}, \bar{b})$, produces $a$ $\exists \wedge \Gamma_{1}$-formula $\psi(\bar{x}, \bar{y})$ such that $\mathfrak{A} \vDash \psi(\bar{a}, \bar{b})$ and $\mathfrak{A} \vDash \forall \bar{y}(\psi(\bar{a}, \bar{y}) \rightarrow \phi(\bar{a}, \bar{y}))$.

In this case the $\exists \wedge \Gamma_{1}$-formulae are existential-positive formulae. Further results may be obtained by applying the theorem to other sets $\Gamma_{1}$ and $\Gamma_{2}$.

THEOREM II. Let $\Gamma$ be an r.e. set with $\left\{P_{i}: i<\omega\right\} \subseteq \Gamma \subseteq \operatorname{Form}(\varrho)$. If $\mathfrak{A}$ is $(\exists \wedge \Gamma) \rightarrow(\exists \wedge \Gamma)$-dec the following are equivalent.

1) Every $\Gamma$-r.e. structure isomorphic to $\mathfrak{A}$ is recursively isomorphic to $\mathfrak{A}$.

2) For some $\bar{a} \subseteq A$ there is an effective procedure which when applied to any $\bar{b} \subseteq A-\bar{a}$ produces $a \exists \wedge \Gamma$-formula $\psi(\bar{a}, \bar{y})$ which is an atom of the Lindenbaum algebra $B(\mathrm{Th}\langle\mathfrak{A}, \bar{a}\rangle)$ such that $\mathfrak{A} \vDash \psi(\bar{a}, \bar{b})$.

Proof. We first show that 2 ) $\Rightarrow 1$ ). Let $\mathfrak{B}$ be a $\Gamma$-r.e. structure and $f: \mathfrak{A} \cong \mathfrak{B}$. We describe by a back and forth argument a rec. map $g: \mathfrak{U} \cong \mathfrak{B}$. At each stage we define sequences $c_{1}, \ldots, c_{n} \in A, d_{1}, \ldots, d_{n} \in B$ and define $g: c_{i} \rightarrow d_{i}$. We ensure that $c_{1}, \ldots, c_{n}$ and $d_{1}, \ldots, d_{n}$ satisfy the same formulae in $\mathfrak{A}$ and $\mathfrak{B}$.

Let $\bar{a}$ be the sequence described in 2). Notice that $\psi(\bar{a}, \bar{y})$ is an atom of $B(\operatorname{Th}\langle\mathscr{A}, \bar{a}\rangle)$ if and only if $\psi(f(\bar{a}), \bar{y})$ is an atom of $B(\operatorname{Th}\langle\mathfrak{B}, f(\bar{a})\rangle)$.

Stage 0 . Define $g(\bar{a})=f(\bar{a})$. Clearly $\bar{a}$, and $g(\bar{a})$ satisfy the same formulae. 
Stage $2_{s+1}$. Suppose we have $g$ defined on $\bar{a}, c_{1}, \ldots, c_{n}$ at this stage by

$$
g\left(\bar{a}, c_{1}, \ldots, c_{n}\right)=f(\bar{a}), d_{1}, \ldots, d_{n} .
$$

Take $c_{n+1}$ to be the least $a \in A-\left\{\bar{a}, c_{1}, \ldots, c_{n}\right\}$. We wish to find $d_{n+1}$. Apply the effective procedure described in 2) to $c_{1}, \ldots, c_{n+1}$ to obtain an atom $\exists \bar{x} \phi\left(\bar{a}, x_{1}, \ldots, x_{n+1}, \bar{x}\right)$ of $B(\operatorname{Th}\langle\mathfrak{U}, \bar{a}\rangle)$ such that $\mathfrak{A} \vDash \exists \bar{x} \phi\left(\bar{a}, c_{1}, \ldots, c_{n+1}, \bar{x}\right)$. Therefore $\mathfrak{A} \vDash \exists \bar{x} x_{n+1} \phi\left(\bar{a}, c_{1}, \ldots, c_{n}, x_{n+1}, \bar{x}\right)$. Since $\bar{a}, c_{1}, \ldots, c_{n}$ and $f(\bar{a})$, $d_{1}, \ldots, d_{n}$ satisfy the same formulae, we therefore have $\mathfrak{B} \vDash$ $\exists \bar{x} x_{n+1} \phi\left(f(\bar{a}), d_{1}, \ldots, d_{n}, x_{n+1}, \bar{x}\right)$. Thus there is a $b \in B$ such that $\mathscr{B}$ F $\exists \bar{x} \phi\left(f(\bar{a}), d_{1}, \ldots, d_{n}, b, \bar{x}\right)$. Since $\mathfrak{B}$ is $\Gamma$-r.e. and therefore $\exists \wedge \Gamma$-r.e., we can find this $b$ effectively. Define $g\left(c_{n+1}\right)=b$, that is, $b=d_{n+1} . \bar{a}, c_{1}, \ldots, c_{n+1}$ and $f(\bar{a}), d_{1}, \ldots, d_{n+1}$ satisfy the same formulae since $\exists \bar{x} \phi\left(\bar{a}, x_{1}, \ldots, x_{n+1}, \bar{x}\right)$ is an atom.

Stage $2_{s+2}$. Suppose we have $g\left(\bar{a}, c_{1}, \ldots, c_{n}\right)=f(\bar{a}), d_{1}, \ldots, d_{n}$. Take $d_{n+1}$ to be the least $b \in B-\left\{f(\bar{a}), d_{1}, \ldots, d_{n}\right\}$. We wish to find $c_{n+1}$. Notice that each sequence $b \subseteq B-f(\bar{a})$ satisfies an atom of $B(\mathrm{Th}\langle\mathfrak{B}, f(\bar{a})\rangle)$; namely the one produced by applying the procedure of 2$)$ to $f^{-1}(\bar{b})$. From an r.e. list of sequences of length $n+1$ from $A-\bar{a}$, produce an r.e. list of atoms of $B(\operatorname{Th}\langle\mathfrak{A}, \bar{a}\rangle)$ by applying the effective procedure of 2 ) to each sequence. As was noticed previously, $d_{1}, \ldots, d_{n+1}$ satisfies one of these. Since $B$ is $\exists \wedge \Gamma$-r.e. we can find effectively the atom $\exists \bar{x} \phi\left(\bar{a}, x_{1}, \ldots, x_{n+1}, \bar{x}\right)$ such that $\mathscr{B}$ F $\exists \bar{x} \phi\left(f(\bar{a}), d_{1}, \ldots, d_{n+1}, \bar{x}\right)$. Since $\bar{a}, c_{1}, \ldots, c_{n}$ and $f(\bar{a}), d_{1}, \ldots, d_{n}$ satisfy the same formulae, arguing as before, there is an $a \in A$ such that $\mathfrak{A} F$ $\exists \bar{x} \phi\left(\bar{a}, c_{1}, \ldots, c_{n}, a, \bar{x}\right) . \mathfrak{A}$ is $\exists \wedge \Gamma$-r.e., and we can therefore find this $a$ effectively. Define $c_{n+1}=a$. Once again, $\bar{a}, c_{1}, \ldots, c_{n+1}$, and $f(\bar{a}), d_{1}, \ldots, d_{n+1}$ satisfy the same formulae.

This process clearly describes a recursive map $g: \mathfrak{A} \cong \mathfrak{B}$.

Notice that in the above proof, at stages $2_{s+1}$ and $2_{s+2}$, we used only the fact that $\bar{a}, c_{1}, \ldots, c_{n}$ and $f(\bar{a}), d_{1}, \ldots, d_{n}$ satisfy the same $\exists \wedge \Gamma$-formulae. We say that a formula $\psi(\bar{a}, \bar{y})$ is an atom of the $\exists \wedge \Gamma$-part of $B(\mathrm{Th}\langle, \bar{a}\rangle)$ if given any $\exists \wedge \Gamma$-formula $\psi(\bar{x}, \bar{y})$ if $\mathfrak{A} \vDash \exists \bar{y}(\psi(\bar{a}, \bar{y}) \wedge \phi(\bar{a}, \bar{y}))$ then $\mathfrak{A} \vDash \forall \bar{y}(\psi(\bar{a}, \bar{y}) \rightarrow$ $\phi(\bar{a}, \bar{y}))$.

Consider the following statement.

$\left.2^{\prime}\right)$ For some $\bar{a} \subseteq A$ there is an effective procedure which when applied to any $\bar{b} \subseteq A-\bar{a}$ produces a $\exists \wedge \Gamma$-formula $\psi(\bar{a}, \bar{y})$ which is an atom of the $\exists \wedge \Gamma$-part of $B(\operatorname{Th}\langle\mathfrak{A}, \bar{a}\rangle)$ such that $\mathfrak{A} \vDash \psi(\bar{a}, \bar{b})$.

Clearly 2$) \Rightarrow 2^{\prime}$ ). We show that $\left.2^{\prime}\right) \Rightarrow 2$ ) by showing that the atoms $\psi(\bar{a}, \bar{y})$ of $\left.2^{\prime}\right)$ are in fact atoms of the whole of $B(\operatorname{Th}\langle\mathfrak{A}, \bar{a}\rangle)$. 
Let $\psi(\bar{a}, \bar{y})$ be an atom of $\left.2^{\prime}\right)$. Let $\bar{a}_{1}, \bar{a}_{2} \subseteq A-\bar{a}$ be such that $\mathfrak{A} \vDash \psi\left(\bar{a}, \bar{a}_{1}\right)$ and $\mathfrak{A} \vDash \psi\left(\bar{a}, a_{2}\right)$. We show that $\psi(\bar{a}, \bar{y})$ is an atom of $B(\operatorname{Th}\langle\mathfrak{A}, \bar{a}\rangle)$ by showing that there is an automorphism $g: \mathfrak{A} \cong \mathscr{A}$ such that $g(\bar{a})=\bar{a}$ and $g\left(\bar{a}_{1}\right)=\bar{a}_{2}$. At stage 0 define $g\left(\bar{a}, \bar{a}_{1}\right)=\bar{a}, \bar{a}_{2}$, and define the map at stages $2_{s+1}$ and $2_{s+2}$ as was done previously.

This shows that 2) $\Leftrightarrow 2^{\prime}$ ).

We now show that $\left.\neg 2^{\prime}\right) \Rightarrow \neg 1$ ) via a finite injury priority construction. Let $\mathfrak{A}$ be a $(\exists \wedge \Gamma) \rightarrow(\exists \wedge \Gamma)$-dec. structure on rec. universe $A=\left\{a_{0}, a_{1}, \ldots\right\}$ satisfying $\left.\neg 2^{\prime}\right)$. We shall construct in stages a $\Gamma$-r.e. $\mathfrak{B}$ on rec. universe $B=\left\{b_{0}, b_{1}, \ldots\right\}$ isomorphic but not recursively isomorphic to $\mathfrak{A}$.

As in Theorem I we shall define at each stage $s$ a partial map $g_{s}: B \rightarrow A$ so that $g=\lim _{s} g_{s}$ is a surjection from $B$ to $A$. We shall also define finite sets $\Sigma^{s}$ and $\Delta^{s}$ of $\exists \wedge \Gamma$-sentences $\psi(\bar{b})$ with $\bar{b} \subseteq B . \mathfrak{B}$ is defined to be the structure on $B$ satisfying all the quantifier-free sentences in $\Sigma=\cup_{s} \Sigma^{s}$. We will ensure that $g: \mathfrak{B} \cong \mathfrak{A}$.

Let $\phi_{0}, \phi_{1}, \ldots$ be a list of all $\exists \wedge \Gamma$-sentences $\phi(\bar{b})$ with $\bar{b} \subseteq B$. Let $f_{0}, f_{1}, \ldots$ be a list of all partial rec. functions from $B$ to $A$. As in Theorem $\mathrm{l}$, the idea is to diagonalize over the $f_{e}: e<\omega$ to ensure that none of them is an isomorphism from $\mathfrak{B}$ to $\mathfrak{A} . f_{e}^{s}$ is the subset of $f_{e}$ computed by stage $s$.

Our requirements are:

$P_{e}^{1}: b_{e} \in \operatorname{dom}(g)$,

$P_{e}^{2}: a_{e} \in \operatorname{ran}(g)$,

$P_{e}^{3}$ : If $\phi_{e}=\phi_{e}(\bar{b})$, say, and $\mathfrak{B} \vDash \phi_{e}(\bar{b})$, then $\phi_{e}(\bar{b}) \in \Sigma$, and

$Q_{e}: f_{e}: \mathfrak{B} \rightarrow \mathfrak{A}$ is not an isomorphism.

Definitions. The four definitions for $P_{e}^{1}$ and $P_{e}^{2}$ are as in Theorem I.

$P_{e}^{3}$ requires attention at stage $s+1$ if $\phi_{e}=\phi_{e}(\bar{b})$ say, has $\bar{b} \subseteq \operatorname{dom}\left(g_{s}\right)$ and $\phi_{e} \notin \Sigma^{s} \cup \Delta^{s}$.

In order to meet the requirements $Q_{e}$ we define at some stages $s$ sequences $\overline{d_{e}^{s}} \subseteq B$ with the intention that if $f_{e}$ is a total function, $\bar{d}_{e}=\lim _{s} \overline{d_{e}^{s}}$ exists, and there is a formula $\theta(\bar{x}) \in \exists \wedge \Gamma$ such that precisely one of $\mathfrak{B} \vDash \theta\left(\vec{d}_{e}\right), \mathfrak{Y} \vDash$ $\theta\left(f_{e}\left(\bar{d}_{e}\right)\right)$ is true.

$Q_{e}$ requires attention at stage $s+1$ if $\overline{d_{e}^{s}}$ is undefined.

$Q_{e}$ is injured at stage $s+1$ if $g_{s+1}\left(\overline{d_{e}^{s}}\right) \neq g_{s}\left(\overline{d_{e}^{s}}\right)$. In this case we say that $\overline{d_{e}^{s+1}}$ is undefined. (Otherwise $\overline{d_{e}^{s+1}}=\overline{d_{e}^{s}}$ )

At any stage $s$ when $P_{0}^{1}, \ldots, P_{e}^{1}, P_{0}^{2}, \ldots, P_{e}^{2}$ do not require attention we define the splitting of $\operatorname{dom}\left(g_{s}\right)$ with respect to $Q_{e}$ as $\operatorname{dom}\left(g_{s}\right)=\bar{b}_{0}, \bar{b}_{1}, \bar{b}_{2}$ where:

$\bar{b}_{0}=b_{0}, b_{1}, \ldots, b_{e}, g_{s}^{-1}\left(a_{0}\right), \ldots, g_{s}^{-1}\left(a_{e}\right), \cup \overline{d_{e^{\prime}}^{s}}($ as in Theorem I); 
$\bar{b}_{1}=\left(\operatorname{dom}\left(f_{e}^{s+1}\right) \cap \operatorname{dom}\left(g_{s}\right)\right)-\bar{b}_{0} ;$ and

$\bar{b}_{2}=\operatorname{dom}\left(g_{s}\right)-\left(\bar{b}_{0} \cup \bar{b}_{1}\right)$.

A $\exists \wedge \Gamma$-sentence $\phi(\bar{b})$ can be used to attack $Q_{e}$ at stage $s+1$ if

1) $\bar{b} \subseteq \operatorname{dom}\left(g_{s}\right)$ and $\bar{b}_{0} \subseteq \operatorname{dom}\left(f_{e}^{s+1}\right)$;

2) $\mathfrak{A} \vDash \exists \bar{x}_{1}, \bar{x}_{2}\left(\wedge \Sigma^{s} \wedge \dot{\phi}\left(g_{s}\left(\bar{b}_{0}\right), \bar{x}_{1}, \bar{x}_{2}\right)\right)$, and

3) $\mathfrak{A} \vDash \neg\left(\forall \bar{x}_{1}\left(\exists \bar{x}_{2} \wedge \Sigma^{s}\left(g_{s}\left(\bar{b}_{0}\right), \bar{x}_{1}, \bar{x}_{2}\right) \rightarrow \exists \bar{x}_{2}\left(\wedge \Sigma^{s} \wedge \phi\left(g_{s}\left(\bar{b}_{0}\right), \bar{x}_{1}, \bar{x}_{2}\right)\right)\right)\right)$ where $\bar{b}_{0}, \bar{x}_{1}, \bar{x}_{2}$ correspond to $\bar{b}_{0}, \bar{b}_{1}, \bar{b}_{2}$ in the splitting of $\operatorname{dom}\left(g_{s}\right)$ with respect to $Q_{e}$.

Construction. Stage 0. Define $g_{0}: b_{0} \rightarrow a_{0} ; \Sigma^{0}=\Delta^{0}=\{\}$.

Stage $s+1$. Arrange the requirements in order of decreasing priority as $P_{0}^{1}, P_{0}^{2}, P_{0}^{3}, P_{1}^{1}, P_{1}^{2}, P_{1}^{3}, \ldots$ and look for the one of highest priority requiring attention at this stage.

If the requirement is a $P_{e}^{1}$ or $P_{e}^{2}$, attack it as was done in Theorem I; and define $\Sigma^{s+1}=\Sigma^{s}$ and $\Delta^{s+1}=\{\}$. If the requirement is a $P_{e}^{3}$, check the requirements $Q_{e^{\prime}}$ with $e^{\prime}<e$ to see if there is one that requires attention at this stage, such that $\phi_{e}$ can be used to attack $Q_{e^{\prime}}$ at this stage. If there is none, attack $P_{e}^{3}$ as follows. (Let $\phi_{e}=\phi_{e}(\bar{b})$.) If $\mathfrak{A} \vDash \phi_{e}\left(g_{s}(\bar{b})\right.$ ), define $\Sigma^{s+1}=\Sigma^{s} \cup\left\{\phi_{e}\right\}$ and $\Delta^{s+1}=\Delta^{s}$. If $\mathfrak{A} \vDash$ $\neg \phi_{e}\left(g_{s}(\bar{b})\right)$, define $\Delta^{s+1}=\Delta^{s} \cup\left\{\phi_{e}\right\}$ and $\Sigma^{s+1}=\Sigma^{s}$. In both cases define $g_{s+1}=$ $g_{s}$.

If there is a $Q_{e^{\prime}}$ with $e^{\prime}<e$, requiring attention such that $\phi_{e}(\bar{b})$ can be used to attack $Q_{e^{\prime}}$ at this stage, then attack $Q_{e^{\prime}}$ as follows.

Case 1. If $\mathfrak{A} \forall \exists \bar{x}_{2}\left(\wedge \Sigma^{s} \wedge \phi_{e}\left(f_{e^{\prime}}^{s+1}\left(\bar{b}_{0}\right), f_{e^{\prime}}^{s+1}\left(\bar{b}_{1}\right), \bar{x}_{2}\right)\right)$, where $\bar{b}_{0}, \bar{b}_{1}, \bar{x}_{2}$ correspond to $\bar{b}_{0}, \bar{b}_{1}, \bar{b}_{2}$ in the splitting of $\operatorname{dom}\left(g_{s}\right)$ with respect to $Q_{e^{\prime}}$, then define $g_{s+1}$ as follows.

$g_{s+1}\left(\bar{b}_{0}\right)=g_{s}\left(\bar{b}_{0}\right)$.

$g_{s+1}\left(\bar{b}_{1}\right)=$ the least sequence $\bar{a}_{1}$ disjoint from $g_{s}\left(\bar{b}_{0}\right)$ such that $\mathfrak{A} \vDash \exists \bar{x}_{2}\left(\wedge \Sigma^{s}\right.$ $\left.\wedge \phi_{e}\left(g_{s}\left(\bar{b}_{0}\right), \bar{a}_{1}, \bar{x}_{2}\right)\right)$. There is such an $\bar{a}_{1}$ by property 2 of $\phi_{e}(\bar{b})$.

$g_{s+1}\left(\bar{b}_{2}\right)=$ the least sequence $\bar{a}_{2}$ disjoint from $g_{s}\left(\bar{b}_{0}\right) \cup \bar{a}_{1}$ such that $\mathfrak{A} \vDash \wedge \Sigma^{s}$ $\wedge \phi_{e}\left(g_{s}\left(\bar{b}_{0}\right), \bar{a}_{1}, \bar{a}_{2}\right)$. There is such an $\bar{a}_{2}$ by definition of $\bar{a}_{1}$.

Case 2. If $\mathfrak{A} \vDash \exists \bar{x}_{2}\left(\wedge \Sigma^{s} \wedge \phi_{e}\left(f_{e^{e^{\prime}}}^{s+1}\left(\bar{b}_{0}\right), f_{e^{\prime}}^{s+1}\left(\bar{b}_{1}\right), \bar{x}_{2}\right)\right)$, define $g_{s+1}$ as follows.

$g_{s+1}\left(\bar{b}_{0}\right)=g_{s}\left(\bar{b}_{0}\right)$.

$g_{s+1}\left(\bar{b}_{1}\right)=$ the least sequence $\bar{a}_{1}$ disjoint from $g_{s}\left(\bar{b}_{0}\right)$ such that $\mathfrak{A} \vDash \exists \bar{x}_{2} \wedge$ $\Sigma^{s}\left(g_{s}\left(\bar{b}_{0}\right), \bar{a}_{1}, \bar{x}_{2}\right) \wedge \neg\left(\exists \bar{x}_{2}\left(\wedge \Sigma^{s} \wedge \phi_{e}\left(g_{s}\left(\bar{b}_{0}\right), \bar{a}_{1}, \bar{x}_{2}\right)\right)\right)$. There is such an $\bar{a}_{1}$ by property 3 of $\phi_{e}(\bar{b})$.

$g_{s+1}\left(\bar{b}_{2}\right)=$ the least sequence $\bar{a}_{2}$ disjoint from $g_{s}\left(\bar{b}_{0}\right) \cup \bar{a}_{1}$ such that $\mathfrak{A} F$ $\wedge \Sigma^{s}\left(g_{s}\left(\bar{b}_{0}\right), \bar{a}_{1}, \bar{a}_{2}\right)$.

In both cases define $\Sigma^{s+1}=\Sigma^{s}, \Delta^{s+1}=\{\}$ and $\overline{d_{e}^{s+1}}=\bar{b}_{0}, \vec{b}_{1}$.

We conclude the proof with the following remarks. 
1) The construction is recursive. The proof is similar to the corresponding proof in Theorem $\mathrm{I}$.

2) Each requirement $Q_{e}$ is attacked at most a finite number of times. The proof is as in Theorem I.

LEMMA 1. $g=\lim _{s} g_{s}$ exists and $g: \mathfrak{B} \cong \mathfrak{A}$.

Proof. Arguing as in Lemma 1 of Theorem I, $g=\lim _{s} g_{s}$ exists and is a surjection from $B$ to $A$.

We show that for every predicate $P$ in $\mathcal{L}, \mathscr{B} \vDash P(\bar{b})$ if and only if $\mathscr{A} \vDash P(g(\bar{b}))$. If $\mathfrak{B} \vDash P(\bar{b})$, just as in Lemma 1 of Theorem I, $\mathfrak{A} \vDash P(g(\bar{b})$ ).

Let $\mathfrak{A} \vDash P(g(\bar{b}))$ and $P(\bar{b})=\phi_{e}$. Consider the stage $s$ at which $g$ takes on its final value on $\bar{b}$. By the construction, since $g_{s} \neq g_{s-1}, \Delta^{s}=\{\}$. Since $\mathfrak{A} \vDash P(g(\bar{b}))$, $P(\bar{b}) \notin \Delta^{t}$ for any $t \geqslant s$. If $P(\bar{b}) \notin \Sigma^{s}$, then $P_{e}^{3}$ requires attention at stage $s+1$, and at some further stage is attacked. In either case $\phi_{e}(\bar{b}) \in \Sigma$, and therefore $\mathfrak{B} \vDash \phi_{e}(\bar{b})$.

LEMMA 2. $\mathfrak{B}$ is a $\Gamma$-r.e. structure.

Proof. By applying to any $\exists \wedge \Gamma$-formula $\phi$ the argument applied to the predicate $P$ above, we see that $\phi(\bar{b}) \in \Sigma$ if and only if $\mathscr{A} \vDash \phi(g(\bar{b})) . g: \mathfrak{B} \cong \mathfrak{A}$; and by Remark $1, \Sigma$ is an r.e. set of $\exists \wedge \Gamma$-sentences $\phi(\bar{b})$. Thus $B$ is a $\exists \wedge \Gamma$-r.e. structure.

LEMMA 3. $\mathscr{B}$ is not recursively isomorphic to $\mathfrak{A}$.

Proof. Suppose $\mathfrak{B}$ is recursively isomorphic to $\mathfrak{A}$. We show that statement $2^{\prime}$ ) is true. Let $e$ by least with $f_{e}: \mathfrak{A} \cong \mathfrak{B}$.

Consider a stage $s_{0}$ after which none of $Q_{0}, \ldots, Q_{e-1}$ are ever attacked and $P_{0}^{1}, \ldots, P_{e}^{1}, P_{0}^{2}, \ldots, P_{e}^{2}$ do not require attention. Let $\bar{b}_{0}$ be as in the splitting of $\operatorname{dom}\left(g_{s_{0}}\right)$ with respect to $Q_{e}$. By the choice of $s_{0}, \bar{b}_{0}$ is the same in the splitting of $\operatorname{dom}\left(g_{s}\right)$ with respect to $Q_{e}$ for any $s \geqslant s_{0}$. Therefore $g_{s_{0}}\left(\bar{b}_{0}\right)=g\left(\bar{b}_{0}\right)=\bar{a}_{0}$, say.

$Q_{e}$ is not injured after stage $s_{0}$. Suppose $Q_{e}$ were attacked at some stage $t \geqslant s_{0}$ by a sentence $\phi(\bar{b})$. Then $\bar{d}_{e}^{t}$ would be defined; and as $Q_{e}$ is never injured, $\bar{d}_{e}=\bar{d}_{e}^{t}$ and $g\left(\bar{d}_{e}\right)=g_{t}\left(\bar{d}_{e}\right)$. Consider the formula $\theta(\bar{x})=\exists \bar{y}\left(\wedge \Sigma^{\prime} \wedge \phi(\bar{x}, \bar{y})\right)$ where $\bar{x}$ corresponds to $\bar{b}_{0}, \bar{b}_{1}$ and $\bar{y}$ to $\bar{b}_{2}$ in the splitting of $\operatorname{dom}\left(g_{t}\right)$ with respect to $Q_{e}$. Since $g_{t}\left(\bar{b}_{0}, \bar{b}_{1}\right)=g\left(\bar{b}_{0}, \bar{b}_{1}\right)$ and $g: \mathfrak{B} \cong \mathfrak{A}$ we have that precisely one of $\mathfrak{B} \vDash \theta\left(\bar{d}_{e}\right)$, $\mathfrak{A} \vDash \theta\left(f_{e}\left(\bar{d}_{e}\right)\right)$ is true. This contradicts the choice of $f_{e}$. Thus $Q_{e}$ is not attacked after stage $s_{0}$. 
Given any $\bar{c} \subseteq B-\overline{b_{0}}$, perform the effective construction up to a stage $s \geqslant s_{0}$ when $\bar{b}_{0} \cup \bar{c} \subseteq \operatorname{dom}\left(g_{s}\right) \cap \operatorname{dom}\left(f_{e}^{s}\right)$. Consider the $\exists \wedge \Gamma$-formula $\psi(\bar{x}, \bar{y})=\exists \bar{z}$ $\wedge \Sigma^{s}(\bar{x}, \bar{y}, \bar{z})$ where $\bar{x}, \bar{y}, \bar{z}$ correspond to $\bar{b}_{0}, \bar{c}$ and $\operatorname{dom}\left(g_{s}\right)-\left(\bar{b}_{0} \cup \bar{c}\right)$ respectively. Clearly $\mathfrak{B} \vDash \psi\left(\bar{b}_{0}, \bar{c}\right)$. We show that $\psi\left(\bar{a}_{0}, \bar{y}\right)$ is an atom of the $\exists \wedge \Gamma$-part of $B\left(\mathrm{Th}\left\langle\mathfrak{H}, \bar{a}_{0}\right\rangle\right)$.

Consider the first stage $m+1>s$ when $\Sigma^{m+1} \neq \Sigma^{s}$; let $\Sigma^{m+1}-\Sigma^{s}=\{\sigma(\bar{b})\}$. By definition of attack on $P^{3}$, this means that $\mathfrak{A} \vDash \sigma\left(g_{m}(\bar{b})\right)$. This implies that the first two conditions in the definition of " $\sigma(\bar{b})$ can be used to attack $Q_{e}$ at stage $m+1$ " are satisfied. However, $Q_{\boldsymbol{e}}$ is not attacked at stage $m+1$. Therefore, condition 3 fails; that is,

$$
\mathfrak{U} \vDash \forall \bar{x}_{1}\left(\exists \bar{x}_{2} \wedge \Sigma^{s}\left(\bar{a}_{0}, \bar{x}_{1}, \bar{x}_{2}\right) \rightarrow \exists \bar{x}_{2}\left(\wedge \Sigma^{s} \wedge \sigma\left(\bar{a}_{0}, \bar{x}_{1}, \bar{x}_{2}\right)\right)\right)
$$

where $\bar{x}_{1}, \bar{x}_{2}$ correspond to $\bar{b}_{1}, \bar{b}_{2}$ in the splitting of $\operatorname{dom}\left(g_{m}\right)$ with respect to $Q_{e}$. Since $\bar{c} \subseteq \bar{b}_{1}$ we may deduce from this that $\mathfrak{A} \vDash \forall \bar{y}\left(\exists \bar{x}^{\prime} \wedge \Sigma^{s}\left(\bar{a}_{0}, \bar{y}, \bar{x}^{\prime}\right) \rightarrow \exists \bar{x}^{\prime} \wedge\right.$ $\left.\Sigma^{m+1}\left(\bar{a}_{0}, y, \bar{x}^{\prime}\right)\right)$ where $\bar{y}, \bar{x}^{\prime}$ correspond to $\bar{c}, \operatorname{dom}\left(g_{m}\right)-\left(\bar{b}_{0} \cup \bar{c}\right)$. Applying this argument repeatedly, we see that for any $t \geqslant s \mathfrak{A} \vDash \forall \bar{y}\left(\exists \bar{x}^{\prime} \wedge \Sigma^{s}\left(\bar{a}_{0}, \bar{y}, \bar{x}^{\prime}\right) \rightarrow\right.$ $\left.\exists \bar{x}^{\prime} \wedge \Sigma^{t}\left(\bar{a}_{0}, \bar{y}, \bar{x}^{\prime}\right)\right)$ where $\bar{y}, \bar{x}^{\prime}$ correspond to $\bar{c}, \operatorname{dom}\left(g_{t}\right)-\left(\bar{b}_{0} \cup \bar{c}\right)$. Call this property $\sharp$.

Consider any $\exists \wedge \Gamma$-formula $\phi\left(\bar{a}_{0}, \bar{y}\right)$ consistent with $\psi\left(\bar{a}_{0}, \bar{y}\right)$. If $\phi\left(\bar{a}_{0}, \bar{c}\right) \in \Sigma^{t}$ for some $t \geqslant s$, by property $\#, \mathfrak{A} \vDash \forall \bar{y}\left(\psi\left(\bar{a}_{0}, \bar{y}\right) \rightarrow \phi\left(\bar{a}_{0}, \bar{y}\right)\right)$. If $\phi\left(\bar{a}_{0}, \bar{c}\right) \notin \Sigma$, then (by virtue of the fact that $\Delta$ is reduced to \{\} at every stage a requirement $P^{1}$ or $P^{2}$ is attacked) there is a stage $t \geqslant s$ at which the $P^{3}$ corresponding to $\phi\left(\bar{a}_{0}, \bar{c}\right)$ is the requirement of highest priority requiring attention. Since $\phi\left(\bar{a}_{0}, \bar{y}\right)$ is consistent with $\psi\left(\bar{a}_{0}, \bar{y}\right)$; by property $\sharp$, the first two conditions in the definition of " $\phi\left(\bar{a}_{0}, \bar{c}\right)$ can be used to attack $Q_{e}$ at stage $t$ " are satisfied. However, $Q_{e}$ is not attacked at stage $t$. Therefore, condition 3 fails. By property \# this implies that $\mathfrak{A} \vDash \forall \bar{y}\left(\psi\left(\bar{a}_{0}, \bar{y}\right) \rightarrow \phi\left(\bar{a}_{0}, \bar{y}\right)\right)$. Thus $\psi\left(\bar{a}_{0}, \bar{y}\right)$ is an atom of the $\exists \wedge \Gamma$-part of $B\left(\operatorname{Th}\left\langle\mathfrak{A}, \bar{a}_{0}\right\rangle\right)$.

We have described an effective procedure which when applied to any $\bar{c} \subseteq B-\bar{b}_{0}$ produces an atom $\psi\left(\bar{a}_{0}, \bar{y}\right)$ of the $\exists \wedge \Gamma$-part of $B\left(\operatorname{Th}\left\langle\mathfrak{A}, \bar{a}_{0}\right\rangle\right)$, such that $\mathfrak{B} \vDash \psi\left(\bar{b}_{0}, \bar{c}\right)$. Notice that any $\bar{d} \subseteq A-\bar{a}_{0}$ satisfies one of these atoms; namely, the one corresponding to $g^{-1}(\bar{d})$. Apply this effective procedure to an r.e. list of all sequences in $B-\bar{b}_{0}$ to obtain an r.e. list of such atoms. Given any $\bar{d} \subseteq A-\bar{a}_{0}$ find an element from this list that $\bar{d}$ satisfies. Since $\mathfrak{A}$ is $\exists \wedge \Gamma$-rec., we can conduct the search effectively.

Thus statement $2^{\prime}$ ) is true, and therefore so is Theorem II.

In the proof of Lemma 3, all that was used to arrive at a contradiction is that for some $e<\omega, B \subseteq \operatorname{dom}\left(f_{e}\right)$ and $f_{e}: \mathfrak{B} \rightarrow f_{e}(\mathscr{B})$ is an isomorphism. Thus we 
actually constructed a $\Gamma$-r.e. $\mathfrak{B}$ isomorphic to $\mathfrak{A}$ but not recursively embeddable in $\mathfrak{U}$.

Under the assumption that 2 ) is false we can in fact construct a countable number of $\Gamma$-r.e. structures $\mathfrak{B}_{0}, \mathfrak{B}_{1}, \ldots$ such that for each $i, \mathfrak{B}_{i} \cong \mathfrak{A}$, and for $i \neq j$, $\mathfrak{B}_{i}$ is not recursively embeddable in $\mathfrak{B}_{j}$.

The $\mathfrak{B}_{i}$ are constructed on universe $B=\left\{b_{0}, b_{1}, \ldots\right\}$ as follows. At each stage $s$ we define, for each $i<\omega$, partial maps $g_{i}^{s}: B \rightarrow A$ and finite sets $\Sigma_{i}^{s}, \Delta_{i}^{s}$ of $\exists \wedge \Gamma$-sentences $\phi(\bar{b})$ with $\bar{b} \subseteq B . \mathfrak{B}_{i}$ is the structure on $B$ satisfying the quantifier-free sentences in $\Sigma_{i} . g_{i}$ will be an isomorphism from $\mathfrak{B}_{i}$ to $\mathfrak{A}$.

Let $f_{0}, f_{1}, \ldots$ be a list of all partial recursive functions from $B$ to $B$.

Our requirements are:

$P_{e, i}^{1}: b_{e} \in \operatorname{dom}\left(g_{i}\right)$,

$P_{e, i}^{2}: a_{e} \in \operatorname{ran}\left(g_{i}\right)$

$P_{e, i}^{3}:$ If $\phi_{e}=\phi_{e}(\bar{b})$ and $B_{i} \vDash \phi_{e}(\bar{b})$, then $\phi_{e}(\bar{b}) \in \Sigma_{i}$, and

$Q_{e, i, j}: f_{e}: \mathfrak{B}_{i} \rightarrow \mathfrak{B}_{j}$ is not an embedding.

The definitions are obtained by appropriate modifications to the previous definitions, as follows.

The splitting of $\operatorname{dom}\left(g_{i}^{s}\right)$ with respect to $Q_{e, i, j}$ is

$\bar{b}_{0}=$ as before,

$\bar{b}_{1}=\left(\operatorname{dom}\left(g_{i}^{s}\right) \cap f_{\underline{e}}^{s+1^{\prime \prime}}\left(\operatorname{dom}\left(g_{j}^{s}\right)\right)\right)-\bar{b}_{0}$, and

$\bar{b}_{2}=\operatorname{dom}\left(g_{i}^{s}\right)-\left(\bar{b}_{0} \cup \bar{b}_{1}\right)$.

Sentence $\phi(\vec{b})$ can be used to attack $Q_{e, i, j}$ at stage $s+1$ if

1) $\vec{b} \subseteq \operatorname{dom}\left(g_{i}^{s}\right)$ and $\bar{b}_{0} \subseteq f_{e}^{s+1^{-1}}\left(\operatorname{dom}\left(g_{j}^{s}\right)\right)$;

2 ) and 3) are obtained from the previous ones by substituting $g_{i}^{s}$ for $g_{s}$.

Impose some order on the requirements and perform the same construction.

Decide if $\mathfrak{A} \vDash \phi_{e}\left(g_{i}^{s}(\bar{b})\right)$ in order to attack $P_{e, i}^{3}$, and distinguish between the two cases in the attack of $Q_{e^{\prime}, i, j}$ by asking if

$\mathfrak{U} \vDash \exists \bar{x}_{2}\left(\wedge \Sigma_{i}^{s} \wedge \phi_{e}\left(g_{j}^{s}\left(f_{e^{\prime}}^{s+1}\left(\bar{b}_{0}, \bar{b}_{1}\right)\right), \bar{x}_{2}\right)\right)$.

The definition of $g_{i}^{s+1}$ is obtained by replacing $g_{s}$ by $g_{i}^{s}$ and $\Sigma^{s}$ by $\Sigma_{i}^{s}$ in the previous definition.

The remarks are proved as in the previous case.

We therefore have

THEOREM II'. If $B$ is $(\exists \wedge \Gamma) \rightarrow(\exists \wedge \Gamma)$-dec., the following are equivalent.

1) Statement 2) of Theorem II is false.

2) There is a $\Gamma$-r.e. structure $\mathfrak{B}$ isomorphic to $\mathfrak{A}$ but not recursively embeddable in $\mathfrak{U}$.

3) There are countably many $\Gamma$-r.e. structures $\mathfrak{B}_{0}, \mathfrak{B}_{1}, \ldots$ isomorphic to $\mathfrak{A}$, such that if $i \neq j, \mathfrak{B}_{i}$ is not recursively embeddable in $\mathfrak{B}_{j}$. 
By applying Theorem II to $\Gamma=$ Form(E), we get

COROLlaRY 2.1 (Nurtazin [6]). For a dec. structure $\mathfrak{A}$ the following are equivalent.

1) $\mathfrak{A}$ is decidably categorical.

2) For some $\bar{a} \subseteq A$ there is an effective procedure which when applied to any $\bar{b} \subseteq A-\bar{a}$ produces a formula $\psi(\bar{a}, \bar{y})$ which is an atom of $B(\mathrm{Th}\langle\mathfrak{A}, \bar{a}\rangle)$, such that $\mathfrak{U} \vDash \psi(\bar{a}, \bar{b})$.

For $\Gamma=\left\{P_{i}: i<\omega\right\} \cup\left\{\neg P_{i}: i<\omega\right\}$ the theorem gives

COROLlaRY 2.2 (Goncharov [3]). For a 2-rec. structure $\mathfrak{A}$ the following are equivalent.

1) $\mathfrak{A}$ is recursively categorical.

2) For some $\bar{a} \subseteq A$ there is an effective procedure which when applied to any $\bar{b} \subseteq A-\bar{a}$ produces an existential formula $\psi(\bar{a}, \bar{y})$ which is an atom of $B(\operatorname{Th}\langle\mathfrak{A}, \bar{a}\rangle)$, such that $\mathfrak{A} \vDash \psi(\bar{a}, \bar{b})$.

The next corollary is new to the literature. For $\Gamma=\left\{P_{i}: i<\omega\right\}$ we get

COROLlaRy 2.3. For a 2-rec. structure $\mathfrak{A}$ the following are equivalent.

1) Every r.e. structure isomorphic to $\mathfrak{A}$ is recursively isomorphic to $\mathfrak{A}$.

2) For some $\vec{a} \subseteq A$ there is an effective procedure which when applied to any $\bar{b} \subseteq A-\bar{a}$ produces an existential-positive $(\exists \wedge \Gamma)$ formula $\psi(\bar{a}, \bar{y})$ which is an atom of $B(\mathrm{Th}\langle\mathfrak{A}, \bar{a}\rangle)$, such that $\mathfrak{A} \vDash \psi(\bar{a}, \bar{b})$.

In this last corollary, the requirement that $\mathfrak{A}$ is 2 -rec. is stronger than is necessary, that is, $(\exists \wedge \Gamma) \rightarrow(\exists \wedge \Gamma)$-decidability.

Further results may be obtained by applying the theorem to other sets $\Gamma$; for example the set of formulae with $n$ alternations of quantifiers.

Consider a structure $\mathfrak{A}$ on $A=\left\{a_{0}, a_{1}, \ldots\right\}$. For each $i<\omega$ define a unary relation $E_{i}$ on $\mathfrak{A}$ by: $\mathfrak{A} \vDash E_{i}\left(a_{j}\right)$ if and only if $a_{i}=a_{j}$.

Let $\Gamma$ be an r.e. set with $\left\{P_{i}: i<\omega\right\} \subseteq \Gamma \subseteq$ Form(E), and $\bar{\Gamma}=\left\{E_{i}: i<\omega\right\}$. We show that if $\mathfrak{A}$ is $\bar{\Gamma}$-r.e., the following are equivalent.

i) Every isomorphism from $\mathfrak{A}$ to a $\Gamma$-r.e. structure is a rec. isomorphism.

ii) For every $\Gamma$-r.e. structure $\mathfrak{B}$ and isomorphism $f: \mathfrak{A} \cong \mathfrak{B}$, the relations $\left\{f E_{i}\right\}$ are uniformly r.e. on $\mathfrak{B}$. 
Proof. Clearly i) $\Rightarrow$ ii).

Let $\mathfrak{B}$ be a $\Gamma$-r.e. structure, and $f: \mathfrak{A} \cong \mathfrak{B}$. For $a_{i} \in A$ we wish to find $f\left(a_{i}\right)$. Use the fact that $f E_{i}$ is r.e. to find $b \in B$ such that $\mathscr{B} \vDash f E_{i}(b)$. This means that $\mathfrak{A} \vDash E_{i}\left(f^{-1}(b)\right)$; that is, $f^{-1}(b)=a_{i}$. Thus $b=f\left(a_{i}\right)$.

Notice that ii) is statement 1 ) of Theorem $I^{\prime}$ for $\Gamma_{1}=\Gamma$ and $\Gamma_{2}=\bar{\Gamma}$. Applying this theorem to $\Gamma$ and $\bar{\Gamma}$ we get

TheOREM III. Let $\Gamma$ be an r.e. set with $\left\{P_{i}: i<\omega\right\} \subseteq \Gamma \subseteq$ Form(E) and $\bar{\Gamma}$ as above. If $\mathfrak{A}$ is $\exists \wedge(\bar{\Gamma} \cup \Gamma)$-rec.; the following are equivalent.

1) Every isomorphism from $\mathfrak{A}$ to a $\Gamma$-r.e. structure is a recursive isomorphism.

2) For some $\bar{a} \subseteq A$ there is an effective procedure which when applied to any $a_{i} \in A-\bar{a}$ produces $a \exists \wedge \Gamma$-formula $\psi(\bar{x}, y)$ such that $\mathfrak{A} \vDash \psi\left(\bar{a}, a_{j}\right)$ if and only if $a_{i}=a_{j}$.

In the case when $\Gamma=\left\{P_{i}: i<\omega\right\} \cup\left\{\neg P_{i}: i<\omega\right\}$, Theorem III gives

CoRollary 3.1 (Ash and Nerode [1], Goncharov [3]). For a 1-rec. structure $\mathfrak{A}$, the following are equivalent.

1) Every isomorphism from $\mathfrak{A}$ to a rec. structure is a rec. isomorphism.

2) For some $\bar{a} \subseteq A$ there is an effective procedure which when applied to any $b \in A-\bar{a}$ produces an existential formula $\psi(\bar{x}, y)$ such that

$$
\mathfrak{A} \vDash \forall(\psi(\bar{a}, y) \leftrightarrow y \equiv b) .
$$

Theorem III may be applied to other sets $\Gamma$ to produce similar results. Some interesting cases are $\Gamma=\left\{P_{i}: i<\omega\right\}$ (to get a result for r.e. structures), and $\Gamma$ the set of formulae with $n$ alternations of quantifiers.

\section{Addendum}

The characterizations presented in the previous sections are all subject to certain decidability assumptions. Ash and Nerode [1] discuss some cases in which the decidability assumption in Theorem I' may be reduced. Goncharov [4] has shown that in the general case these decidability assumptions cannot be completely removed.

Theorem II has been discussed in particular cases. LaRoche proved that a recursive Boolean algebra is recursively categorical if and only if it has a finite number of atoms. (A proof of this result may be found in Remmel [7].) Remmel [8] showed that a recursive linear order is recursively categorical if and only if it 
has a finite number of successivities. These conditions are equivalent, in these cases, to statement 2) of Theorem II. However, the results do not follow from Theorem II as there are linear orders and Boolean algebras which do not have any structures in their isomorphism type with the decidability required in Theorem II. Goncharov and Dzgoev [2] have generalized these results to produce a condition (branching) sufficient for a rec. structure not to be recursively categorical. Goncharov [5] uses this condition to characterize recursively categorical Abelian $p$-groups, and a similar condition to characterize recursively categorical structures in a language consisting only of unary predicates.

\section{References}

[1] C. Ash and A. Nerode, 'Intrinsically recursive relations', Aspects of effective algebra, pp. 26-41. edited by J. N. Crossley (Upside Down A Book Company, Steel's Creek, Australia, 1981).

[2] V. D. Dzgoev and S. S. Goncharov, 'Autostability of models', Algebra and Logic 19 (1980), $28-37$.

[3] S. S. Goncharov, 'Autostability and computable families of constructivizations', Algebra and Logic 14 (1975), 392-409.

[4] S. S. Goncharov, 'On the number of nonautoequivalent constructivizations', Algebra and Logic 16 (1977), 169-185

[5] S. S. Goncharov, 'Autostability of models and Abelian groups', Algebra and Logic 19 (1980), $13-27$.

[6] A. T. Nurtazin, 'Strong and weak constructivizations and computable families', Algebra and Logic 13 (1974), 177-184.

[7] J. Remmel, 'Recursive isomorphisms of recursive Boolean algebras', J. Symbolic Logic $\mathbf{4 6}$ (1981), 572-594.

[8] J. Remmel, 'Recursively categorical linear orderings', Proc, Amer. Math. Soc. 83 (1981). 379-386.

Department of Mathematics

Monash University

Clayton, Victoria 3158

Australia 